\title{
WHITEHEAD GROUPS OF SEMIDIRECT PRODUCTS OF FREE GROUPS II
}

\author{
by KOO-GUAN CHOO
}

(Received 27 November, 1978)

Let $G$ be a group. We denote the Whitehead group of $G$ by Wh $G$ and the projective class group of the integral group ring $\mathbb{Z}(G)$ of $G$ by $\tilde{K}_{0} \mathbb{Z}(G)$. For undefined terminologies used in the paper, we refer to [3] and [6].

First we recall the definition of semidirect product of groups. Let $G$ be a group, $\alpha$ an automorphism of $G$ and $F$ a free group generated by $\left\{t_{\lambda}\right\}_{\lambda \in \Lambda}$. If $w$ is a word in $t_{\lambda}$ defining an element of $F$, we denote by $|w|$ the total exponent sum of the $t_{\lambda}$ appearing in $w$. The semidirect product $G \times{ }_{\alpha} F$ of $G$ and $F$ with respect to $\alpha$ is defined as follows: $G \times{ }_{\alpha} F=$ $G \times F$ as sets and multiplication in $G \times{ }_{\alpha} F$ is given by

$$
(g, w)\left(g^{\prime}, w^{\prime}\right)=\left(g \alpha^{-|w|}\left(g^{\prime}\right), w w^{\prime}\right),
$$

for any $(g, w),\left(g^{\prime}, w^{\prime}\right)$ in $G \times{ }_{\alpha} F$. In particular, if $F$ is an infinite cyclic group $T=\langle t\rangle$ generated by $t$, we have the semidirect product $G \times{ }_{\alpha} T$ of $G$ and $T$ with respect to $\alpha$.

Next we recall the following definition of a group of type $n$ [6, p. 214]: Any group possessing only a single element will be called a group of type 0 . Inductively, we define $G$ to be a group of type $n$ if $G=H \times{ }_{\alpha} T$ where $H$ is a group of type $n-1$. In particular, any free abelian group of finite rank $n$ is a group of type $n$.

Now let $G$ be a group of type $n, F_{1}, F_{2}, \ldots$ a set of free groups, each of rank at least two, and let

$$
H_{\mathrm{k}}=G \times F_{1} \times \ldots \times F_{k}
$$

be the direct product of $G, F_{1}, \ldots, F_{k}(k=1,2, \ldots)$. Let $\alpha$ be an automorphism of $H_{k}$ which leaves all but one of the $F_{j}(j=1, \ldots, k)$ pointwise fixed. Then, in [5], we have shown:

THEOREM 1 [5, Theorem 1]. For each $k=1,2, \ldots$,

$$
\text { Wh } H_{k}=0, \quad \tilde{K}_{0} \mathbb{Z}\left(H_{k}\right)=0 \text { and } \tilde{C}\left(\mathbb{Z}\left(H_{k}\right) \text {. id }\right)=0 .
$$

TheOREM 2 [5, Theorem 2]. For each $k=1,2, \ldots$,

$$
\mathrm{Wh}\left(H_{k} \times{ }_{\alpha} T\right)=0, \quad \tilde{K}_{0} \mathbb{Z}\left(H_{k} \times{ }_{\alpha} T\right)=0 \quad \text { and } \quad \tilde{C}\left(\mathbb{Z}\left(H_{k} \times{ }_{\alpha} T\right), \text { id }\right)=0,
$$

where $T$ is any infinite cyclic group.

Let $F=\left\langle t_{\lambda}\right\rangle_{\lambda \in \Lambda}$ be another free group and $H_{k} \times_{\alpha} F$ the semidirect product of $H_{k}$ and $F$ with respect to $\alpha(k=1,2, \ldots)$. Then the purpose of this paper is to extend these results to:

MAIN THEOREM. For each $k=1,2, \ldots$,

$$
\mathrm{Wh}\left(H_{k} \times{ }_{\alpha} F\right)=0, \quad \tilde{K}_{0} \mathbb{Z}\left(H_{k} \times_{\alpha} F\right)=0 \quad \text { and } \quad \tilde{C}\left(\mathbb{Z}\left(H_{k} \times{ }_{\alpha} F\right), \text { id }\right)=0 .
$$

Glasgow Math. J. 21 (1980) 71-74. 
Remark. The results clearly extend those of [3]. Moreover, since the group $D$ defined in [4] is just one of our $H_{k}$, these results also give an affirmative answer to the question mentioned at the end of [4].

As noted in [4, p. 29], we have for $k=1,2, \ldots$,

$$
H_{k} \times{ }_{\alpha} F \cong\left(H_{k} \times F^{\prime}\right) *_{H_{k}}\left(H_{k} \times{ }_{\alpha} T\right) \text {, }
$$

where $T=\left\langle t_{\lambda_{0}}\right\rangle$ for some $\lambda_{0}$ and $F^{\prime}$ is the free group generated by $\left\langle t_{\lambda}^{-1} t_{\lambda_{0}} \mid \lambda \neq \lambda_{0}\right\rangle$.

The following direct sum decomposition for free product of groups with amalgamation is due to Waldhausen (cf. $[\mathbf{9}, \S 5]$ or $[\mathbf{1 0}]$ ).

THEOREM 3. Let $A$ and $B$ be two groups and $C$ a common subgroup of $A$ and $B$. Let $A{ }_{C} B$ be the free product with amalgamation and $\gamma: C \rightarrow A, \beta: C \rightarrow B$ the inclusions defining $A{ }_{C} B$. Then there is a natural direct sum splitting for $\mathrm{Wh}\left(A{ }_{C} B\right)$ :

$$
\mathrm{Wh}\left(A{ }_{C} B\right) \cong \mathrm{Wh}(A, B ; C) \oplus \tilde{K}_{0}(C ; A, B) \oplus \tilde{C}(C ; A, B),
$$

where $\mathrm{Wh}(A, B ; C)$ and $\tilde{K}_{0}(C ; A, B)$ are given by the following exact sequences

$$
\begin{aligned}
& \text { Wh } C \stackrel{\gamma_{*}^{\oplus-\beta_{*}}}{\longrightarrow} \text { Wh } A \oplus \mathrm{Wh} B \longrightarrow \mathrm{Wh}(A, B ; C) \longrightarrow 0 ; \\
& 0 \longrightarrow \tilde{K}_{0}(C ; A, B) \longrightarrow \tilde{K}_{0} \mathbb{Z}(C) \stackrel{\gamma_{*} \oplus-\beta_{*}}{\longrightarrow} \tilde{K}_{0} \mathbb{Z}(A) \oplus \tilde{K}_{0} \mathbb{Z}(B) ;
\end{aligned}
$$

and $\tilde{C}(C ; A, B)$ is given in [9, p. 2.2] (we refer the readers to [9] for a detailed description).

Moreover, if $C$ is such that $\mathbb{Z}(C)$ is coherent and of finite global dimension, then the exotic summand $\tilde{C}(C ; A, B)=0$, so that in this case,

$$
\mathrm{Wh}\left(A *_{C} B\right) \cong \mathrm{Wh}(A, B ; C) \oplus \tilde{K}_{0}(C ; A, B) .
$$

Finally, we recall

THEOREM 4 [5, Theorem 4]. Let $G$ be a group of some particular form such that $G$ and $G \times T$ are of the same form, where $T$ is any infinite cyclic group. Suppose that the Whitehead group of any such group is trivial. Let $H_{k}^{\prime}=G \times F_{1} \times \ldots \times F_{k}$, where $F_{1}, \ldots, F_{k}$ are free groups each of rank at least two. Then, for each $k=1,2, \ldots$,

$$
\text { Wh } H_{k}^{\prime}=0, \quad \tilde{K}_{0} \mathbb{Z}\left(H_{k}^{\prime}\right)=0 \quad \text { and } \quad \tilde{C}\left(\mathbb{Z}\left(H_{k}^{\prime}\right), \text { id }\right)=0 .
$$

Proof of the main theorem. First, we prove the theorem for $k=1$. As noted in (2), we have,

$$
H_{1} \times{ }_{\alpha} F \cong\left(H_{1} \times F^{\prime}\right) *_{H_{1}}\left(H_{1} \times_{\alpha} T\right),
$$

where $T=\left\langle t_{\lambda_{0}}\right\rangle$ for some $\lambda_{0}$ and $F^{\prime}=\left\langle t_{\lambda}^{-1} t_{\lambda_{0}} \mid \lambda \neq \lambda_{0}\right\rangle$.

We have pointed out in [5] that if $G$ is a group of type $n$, then $H_{1}=G \times F_{1}$ is coherent and of finite global dimension (cf. [6, Theorem 25] and [2, 7]). Thus the exotic summand $\tilde{C}\left(H_{1} ; H_{1} \times F^{\prime}, H_{1} \times{ }_{\alpha} T\right)=0$ and so the Waldhausen direct sum decomposition for $\mathrm{Wh}\left(H_{1} \times{ }_{\alpha} F\right)$ becomes

$$
\mathrm{Wh}\left(H_{1} \times{ }_{\alpha} F\right) \cong \mathrm{Wh}\left(H_{1} \times F^{\prime}, H_{1} \times{ }_{\alpha} T ; H_{1}\right) \oplus \bar{K}_{0}\left(H_{1} ; H_{1} \times F^{\prime}, H_{1} \times_{\alpha} T\right)
$$


and the following sequences are exact:

$$
\begin{gathered}
\text { Wh } H_{1} \rightarrow \mathrm{Wh}\left(H_{1} \times F^{\prime}\right) \oplus \mathrm{Wh}\left(H_{1} \times{ }_{\alpha} T\right) \rightarrow \mathrm{Wh}\left(H_{1} \times F^{\prime}, H_{1} \times{ }_{\alpha} T ; H_{1}\right) \rightarrow 0 ; \\
0 \rightarrow \tilde{K}_{0}\left(H_{1} ; H_{1} \times F^{\prime}, H_{1} \times{ }_{\alpha} T\right) \rightarrow \tilde{K}_{0} \mathbb{Z}\left(H_{1}\right) \rightarrow \tilde{K}_{0} \mathbb{Z}\left(H_{1} \times F^{\prime}\right) \oplus \tilde{K}_{0} \mathbb{Z}\left(H_{1} \times{ }_{\alpha} T\right) .
\end{gathered}
$$

But $\mathrm{Wh}\left(H_{1} \times F^{\prime}\right)=0, \tilde{K}_{0} \mathbb{Z}\left(H_{1}\right)=0$ by Theorem 1 and $W h\left(H_{1} \times{ }_{\alpha} T\right)=0$ by Theorem 2 . Thus $\mathrm{Wh}\left(H_{1} \times F^{\prime}, H_{1} \times{ }_{\alpha} T ; H_{1}\right)=0$ and $\tilde{K}_{0}\left(H_{1} ; H_{1} \times F^{\prime}, H_{1} \times{ }_{\alpha} T\right)=0$. Hence $W h\left(H_{1} \times\right.$ $\left.{ }_{\alpha} F\right)=0$ and this proves the theorem for $k=1$.

Next, since $G$ is a group of type $n, G \times T$ is a group of type $n+1$. Thus $G$ and $G \times T$ are of the same form and so $H_{1} \times{ }_{\alpha} F=\left(G \times F_{1}\right) \times{ }_{\alpha} F$ and $\left(H_{1} \times{ }_{\alpha} F\right) \times T=\left(G \times T \times F_{1}\right) \times{ }_{\alpha} F$ are of the same form. We have shown above that the Whitehead group of any such group $H_{1} \times_{\alpha} F$ is trivial. Hence the assertions follow immediately from Theorem 4.

This completes the proof.

In addition to the results in the main theorem, we see that

$$
\tilde{C}\left(H_{k} ; H_{k} \times F^{\prime}, H_{k} \times{ }_{\alpha} T\right)=0 \quad(k=2,3, \ldots),
$$

by Theorem 3, although $\mathbb{Z}\left(H_{k}\right)$ is not coherent (cf. [7, p. 42]).

We conclude the paper by the following remark.

Remark. Let $H_{k}, \alpha$ and $F$ be as given above. Let $\mathscr{A}=\left\{\alpha^{n_{\lambda}}\right\}_{\lambda \in \Lambda}$ a set of automorphisms $\alpha^{n_{\lambda}}$ of $H_{k}$, where each $n_{\lambda}$ is an integer, for all $\lambda \in \Lambda$. Let $H_{k} \times{ }_{\mathscr{A}} F$ be the semidirect product of $H_{k}$ and $F$ with respect to $\mathscr{A}$ (cf. [5]). Then a slight modification of arguments, as given above, will give

$$
\mathrm{Wh}\left(H_{k} \times{ }_{\mathscr{A}} F\right)=0, \quad \tilde{K}_{0} \mathbb{Z}\left(H_{k} \times{ }_{\mathscr{A}} F\right)=0 \quad \text { and } \quad \tilde{C}\left(\mathbb{Z}\left(H_{k} \times{ }_{\mathscr{A}} F\right), \text { id }\right)=0 .
$$

\section{REFERENCES}

1. H. Bass, A. Heller and R. G. Swan, The Whitehead group of a polynomial extension, Inst. Hautes Études Sci. Publ. Math. No. 22(1964), 61-79. MR 30\#4806.

2. K. G. Choo, K. Y. Lam and E. Luft, On free product of rings and the coherence property, Algebraic K-theory II: "Classical" algebraic K-theory and connections with arithmetic, Lecture Notes in Mathematics 342 (Springer, 1973), 135-143. MR50\#13154.

3. K. G. Choo, The projective class group of the fundamental group of a surface is trivial, Proc. Amer. Math. Soc. 40(1973), 42-46. MR48\#2222.

4. K. G. Choo, Whitehead groups of certain semidirect products of free groups, Proc. Amer. Math. Soc. 43(1974), 26-30. MR49\#2890.

5. K. G. Choo, Whitehead groups of semidirect products of free groups, Glasgow Math. J., 19(1978), 155-158.

6. F. T. Farrell and W. C. Hsiang, A formula for $K_{1} R_{\alpha}[T]$, Proc. Sympos. Pure Math., vol. 17, (Amer. Math. Soc., 1970), 192-218. MR41\#5457.

7. S. M. Gersten, K-theory of free rings, Comm. Algebra 1(1974), 39-64.

8. J. Stallings, Whitehead torsion of free products, Ann of Math. 82(1965), 354-363. MR31\#3518.

9. F. Waldhausen, Whitehead groups of generalized free products, Preliminary Report (1969). 
10. F. Waldhausen, Whitehead groups of generalized free products, Algebraic K-theory "Classical" algebraic K-theory and connections with arithmetic, Lecture Notes in Mathemat 342 (Springer, 1973), 155-179.

Department of Pure Mathematics

UNIVERSITY OF SYDNEY

SYDNEY, N.S.W. 2006

Australia. 